\title{
Does a live performance impact synchronization to musical rhythm in cognitively impaired elderly?
}

\author{
Matthieu Ghilain $^{1 *}$, Lise Hobeika ${ }^{1,2^{*}}$, Micheline Lesaffre ${ }^{3}$, Loris Schiaratura ${ }^{1}$, Ashmita Singh ${ }^{4}$, Joren \\ $\mathrm{Six}^{3}$, Dominique Huvent-Grelle ${ }^{5}$, François Puisieux ${ }^{5}$, and Séverine Samson ${ }^{1,6}$ \\ ${ }^{1}$ Université de Lille, URL 4072 - PSITEC - Psychologie: Interactions, Temps, Emotions, Cognition, F-59000 Lille, \\ France \\ ${ }^{2}$ Sciences et Technologies de la Musique et du Son, IRCAM, CNRS, Sorbonne Université, F-75004 Paris, France \\ ${ }^{3}$ IPEM, Department of Arts, Music and Theater Sciences, Ghent University, Belgium \\ ${ }^{4}$ Faculty of Medicine, University of Toronto, Canada \\ ${ }^{5}$ Hôpital Gériatrique les Bateliers, Pôle de Gérontologie, CHU Lille, 23 rue des Bateliers, F-59037 Lille France \\ ${ }^{6}$ AP-HP, GHU Pitié-Salpêtrière-Charles Foix, F-75013 Paris, France \\ * these authors contribute equally to this work \\ Correspondence to: severine.samson@univ-lille.fr
}

This is a draft. The published version is available at https://doi.org/10.3233/jad-200521

\begin{abstract}
Background

Music-based interventions appear to be efficient non-pharmacological approaches to improve emotional, social, and cognitive functioning of patients with neurodegenerative diseases such as Alzheimer's Disease (AD).

Objectives

Because the benefits seem to increase with patients motor involvement, we studied the ability to move in synchrony with musical rhythms and more specifically the impact of live performance compared to video recording of a musician on sensorimotor abilities in patients with cognitive impairments (AD, vascular and mixed dementia) and in patients with no evidence of cognitive impairments.

\section{Methods}

Sensorimotor synchronization (SMS) to a metronomic or a musical stimulus was assessed in patients when they were viewing a live musician or his pre-recorded video.

Results

Participants were better at synchronizing to a metronome than to music but this effect was modulated by the social context. While SMS to a metronome was better when facing a video than a live performance, there was no impact of the social context on SMS to music. Lastly, we found no group differences on SMS.

Conclusion

The decrease in synchronization ability to metronome in the live performance may be due to the social pressure associated with the presence of the musician. Such a pressure might be removed in pleasant and ecological social activities, like moving with music, explaining the lack of the social context effect on SMS to music. Remarkably, the group with cognitive impairments performed no worse than the group without cognitive impairments, which suggests relatively spared SMS abilities in these patients. By showing that it is possible to encourage a patient to synchronize with others, even in front of a video, the results of our study indicate that SMS can be used as a relevant predictor in clinical trials and open up promising therapeutic options for patients living in remote areas.
\end{abstract}

Keywords: Alzheimer's disease, Dementia, Music therapy, Social interaction, Motor activity, Aging, Cognitive impairment 


\section{Introduction}

Alzheimer's disease (AD) is a neurodegenerative disorder affecting several aspects of cognition, with symptoms such as memory dysfunction [1], language impairment [2,3], emotional deficits and social withdrawal [4]. Due to the limited efficacy of pharmacological treatments, health institutions have encouraged the development of nonpharmacological approaches in the management of dementia [5]. In parallel, the use of music-based interventions for patients with $\mathrm{AD}$ and dementia has increased over the last decade [6,7] due to their positive impact on patients' emotional, social and cognitive functioning, as well as on caregiver well-being $[8,9]$. Musical interventions can cover a large variety of activities from passive music listening in isolation to active music making in groups. Some studies reported that encouraging motor involvement in patients, by making them move with the musical beats or sing, provides greater benefits than passive music listening [10,11]. Such a finding suggests that motor coordination, and more precisely rhythmic sensorimotor synchronization (SMS), induced by these activities might contribute to the efficacy of music-based interventions in patients with dementia.

A central factor of these musical interventions is the social context: patients generally listen to music in the presence of others (i.e. in a group or in the presence of a musician). It has been demonstrated that the presence of a live musician while listening to music increases patients' spontaneous motor behaviors, compared with listening alone to an audio [12-14] or a video [15] recording of a musical piece. Moreover, previous findings have shown that social presence can modulate SMS. When two individuals perform rhythmic movements together, they tend to spontaneously synchronize their movements $[16,17]$. By coupling their rhythmic movements with oneanother, individuals improve their SMS performances [1820]. In a previous study, we investigated the impact of social context on SMS to musical rhythms as well as on patients' socio-emotional and motor engagement during musical activities in patients with AD [15]. Each participant was asked to tap with a metronomic or a musical rhythm, in the presence of a musician who performed the task. The presence of the musician was real (live condition) or virtual (video condition). Contrary to our predictions, we found that patients' SMS was better when they viewed a video of a musician performing rather than in a face-to-face live performance with a musician. We interpreted this finding by accounting for the effect of social pressure arising from the presence of an observer. Since the patients involved in our previous study all presented with moderate to severe dementia (Alzheimer's Disease, vascular or mixed dementia), and control participants without cognitive impairment were not tested, it remained difficult to determine whether or not the impact of social pressure was related to or exacerbated by the progression of the disease, and by cognitive decline. However, this issue deserves to be further investigated in elderly patients with different levels of cognitive impairments.

Rhythmic SMS requires core cognitive skills such as anticipation, adaptation and attention [21], as well as accurate sensorimotor control. Although spontaneous rhythmic movements are frequently observed, SMS has been rarely investigated in patients with neurodegenerative disease such as AD. Since most studies have explored deficits in cognition, little is known about the motor disorders associated with $\mathrm{AD}$ or other types of dementia $[22,23]$. The few studies that have examined rhythmic tapping abilities in $\mathrm{AD}$ have used continuous metronomic tapping tasks $[24,25]$. In these motor tasks, which are more cognitively challenging than SMS tasks, patients start by tapping with metronome but are required to continue reproducing the metronome even after its removal. Results have shown a decrease in tapping regularity and accuracy in patients with $\mathrm{AD}$, even at the early stage of the disease, as compared with matched elderly participants. However, the origins and the specificity of this decline in performance are not known.

When evaluating SMS, generally two types of sound sequences are used: metronome or musical sequences. Whereas the beat is directly perceived with a metronome, it has to be extracted from the complex auditory patterns in music. It is therefore harder to synchronize with music than with metronome beats $[26,27]$ even though tapping with music is more motivating and enjoyable. Additionally, SMS can be characterized by two complementary measures: consistency (i.e. tap regularity), and asynchrony (i.e. tap precision or anticipation). In the field of neurodegenerative pathologies, data reported in the literature has shown that SMS with music in patients with Parkinson's disease is less consistent and less anticipated than in controls [28-30]. No study, to our knowledge, has compared SMS in patients with AD and in controls.

In line with our previous study, the present one aimed to investigate the impact of social context on SMS to musical rhythms in a different group of cognitively impaired elderly patients (CI group) and compare their performances to elderly participants with no cognitive impairments (NoCI group). The novelty of our paradigm lies in our ability to manipulate the social contexts, in which the task was performed. For this purpose, we used a simple paced tapping task, where participants synchronized tapping to beats in an auditory sequence. While performing the task, each participant viewed a musician who was also tapping synchronously to the auditory sequence, but this occurred in two distinct social environments. In one, participants were privy to a live performance by the musician, leading to face-to-face interactions (live condition). In the other condition, participants were shown the musician's pre-recorded performance projected on a life-sized screen (video condition). Furthermore, auditory context was manipulated by examining SMS in two types of auditory sequences: a metronomic and a musical sequence. These were analyzed based on the participants' consistency and asynchrony. We also measured the participants' spontaneous tapping in 
order to have an independent evaluation of their production abilities. For this task, participants were instructed to tap as regularly as possible at a comfortable rate. In line with previous findings, we expected SMS to be more consistent and more anticipated with metronome than with music, and in video than in live condition. We predicted that SMS in the CI group will be globally less consistent and anticipated than in the NoCI group; and consequently, based on the social cognition deficits associated with cognitively impaired elderly, we hypothesized that any interaction of social and auditory contexts may be more pronounced in participants with no cognitive impairment.

\section{Materials and Methods}

\section{Participants.}

Ninety-seven participants were recruited at the Bateliers Day Hospital (Lille University Hospital, France) in the context of their scheduled medical consultations (following memory or fall problems). All participants were right-handed, and native French speakers. A group of 48 patients with cognitive impairments (CI group) including patients with mild to moderate $\mathrm{AD}(\mathrm{n}=12)$, patients with mixed dementia $(n=30)$ and patients with vascular dementia, $(n=6)$ participated in this study. The diagnosis of Alzheimer's disease (AD), vascular or mixed dementia was based on the Diagnostic and Statistical Manual of Mental Disorders-V criteria (American Psychiatric Association, 2013) and made by a geriatrician. Their results were compared to those of a group of 49 matched patients with no sign of cognitive impairments at the time of testing (NoCI group).

Demographic data encompassing age, gender, sociocultural level [32], musical expertise [33], as well as clinical information, including State-Trait Anxiety Inventory [34], Activities of Daily Living [35], and MiniMental State Examination [36] for both groups are presented in Table 1. The study was approved by the Comité de Protection des Personnes (CPP 18/012) and the Commission Nationale de l'Informatique et des Libertés (CNIL).

\section{Apparatus}

The experimental device consisted of two chairs facing each other. A tablet equipped with a force sensor was attached to the right armrest of each chair, allowing the participant and the musician to perform the SMS task by tapping on the tablet with a ball-like probe in their hands (Figure 1.A). Two social contexts were tested. In the video condition, the musician appeared in front of the participant on a life-size screen $(158 \times 92 \mathrm{~cm})$ at a distance of $215 \mathrm{~cm}$ (Figure 1.B). In the live condition, the musician was facing the participant at a distance of $200 \mathrm{~cm}$ (Figure 1.C). The experimenter was hidden behind a curtain in both social contexts.
Two 60-second auditory sequences were used: a metronomic and a musical sequence, both presented at the same tempo (inter-onset interval (IOI) of $800 \mathrm{~ms}$, corresponding at 75 beats per minutes (bpm)). The metronomic sequence was composed of regular beats. The musical sequence was a musical excerpt from a French folk song ("La Java Bleue"), very well known to the elderly as attested by the participants themselves, with a ternary metric. Four initial beats preceded each auditory sequence to give the tempo.

\section{Design and procedure}

Each participant was tested individually. First, participants performed a spontaneous motor task, by tapping as regularly as possible for 30 taps at a comfortable tempo. Then, participants performed the SMS task by tapping at every beat for all the auditory sequences. The musician also tapped on his tablet at every beat of the auditory sequences, in both, live and video conditions. In addition, he pronounced "ta" at each beat of the metronome sequence and sang the lyrics of the song in the music sequence, with the exact same song excerpts being used during live and video conditions. The participant and the musician performed the SMS task together. The experimental design of the SMS task included one between-subject factor: GROUP (CI / NoCI); and two within-subject factors: AUDIO (Metronomic/Musical) and SOCIAL CONTEXT (Video/Live). The order of presentation of the four experimental conditions was counterbalanced.

\section{Data analysis}

The analysis of the spontaneous motor tapping was performed on 20 taps (the first eight taps and the last two taps being discarded). The mean tapping rate (mean intertap interval, ITI) and the tapping variability (coefficient of variation $(\mathrm{CV})$ of the ITI) were calculated.

SMS measures were analyzed on 64 beats, from a 60 -second auditory sequence for each condition (providing 256 measures by participant), using circular statistics [37] with the CircStat Matlab Toolbox [38], which is a robust tool to quantify SMS when there is not a perfect match between the number of taps and the pacing stimuli [19]. Thus, it is appropriate when participants tend to miss taps or to tap more than once in response to one beat.

Each tap was represented on a circular scale going from $-180^{\circ}$ to $+180^{\circ}$, representing the $800 \mathrm{~ms}$ IOI (see Figure 2). The beat's occurrence corresponds to $0^{\circ}$ : it is the time at which participant was expected to tap. Taps were placed on the circular scale depending on their time delay in relation with the beats. For example, a tap occurring $200 \mathrm{~ms}$ after a beat is represented by a unit vector with an angle of $+90^{\circ}$, whereas a tap occurring 200 $\mathrm{ms}$ before a beat corresponds to a unit vector with an angle of $-90^{\circ}$. All vectors were averaged to obtain a mean resultant vector $\vec{R}[37,38]$ allowing for the calculation of 
synchronization consistency (i.e. regularity) and the accuracy. Vector $\vec{R}$ length indicated the consistency between 0 and 1 , with 1 corresponding to perfect consistency (all taps occurred at the same delay to the beat) and 0 corresponding to an absence of synchronization (the taps were randomly distributed between the beats). Accuracy was calculated only if participant's taps were not randomly distributed. Random distribution of taps was tested using the Rayleigh test for circular uniformity [37,39]. Accuracy was determined based on the angular deviation (' $\theta$ ') of vector $\vec{R}$ from zero, which was then transformed back into milliseconds.

The musician's synchronization performance was checked on the basis of the same 64 beats. The consistently good and proper performances by the musician did not justify the use of circular statistics. The accuracy and variability of synchronization were calculated by the mean and the $\mathrm{CV}$ of asynchronies expressed in milliseconds.

For each analysis, distribution normality was verified using the Kolmogorov-Smirnov test. Wilcoxon Mann-Whitney U t-test, a non-parametric test, was used in cases where the distribution was not normal. The Pearson's Chi-squared test was used for categorical variables (gender and socio-cultural level ratios). The sample effect size for ANOVA was estimated with the partial eta-squared $\left(\eta^{2} p\right)$.

\section{Results}

\section{Preliminary analyses}

\section{Demographic and clinical data}

We compared demographic and clinical data of the CI and NoCI groups. As summarized in Table 1, the analyses showed no group difference in terms of age $(t(95)=1.74$, $p=.08)$, gender (Chi-squared Pearson test : $\chi^{2}(1, \mathrm{~N}=97)$ $=.18, p=.67)$, socio-cultural level (Chi-squared Pearson test : $\left.\chi^{2}(3, \mathrm{~N}=97)=3.38, p=.34\right)$, musical expertise (Mann-Whitney $U$ test : $U=1075, p=.46$ ), and mood assessed with the STAI mood questionnaire (MannWhitney $U$ test : $U=930, p=0.08)$. In contrast, the two groups differed in the severity of cognitive impairment as assessed by the MMSE (Mann-Whitney $\mathrm{U}$ test: $U=0, p$ $<.001$ ), and in functional status as assessed by ADLs (Mann-Whitney U test : $U=543, p<.001$ ).

\section{Spontaneous motor tapping task}

Group comparison revealed no difference in ITIs and CV, indicating that pace and variability did not differ between groups (Mann-Whitney $\mathrm{U}$ test: $p>.05$ in both cases) during spontaneous motor tapping (see Figure 3).

\section{Sensorimotor synchronization task}

By using the Rayleigh test, we first verified whether participants tapped in synchrony with the beat or at random. All the tests were significant except for one NoCI participant who had a non-significant Rayleigh test in one condition, suggesting that this participant did not succeed at synchronizing the taps to the beat in this case. Since this occurred in only one condition for the participant and not in the other conditions, this specific value was replaced by the group average for this condition.

SMS Consistency. We conducted an ANOVA on SMS consistency with the factors AUDIO (metronome/music), SOCIAL CONTEXT (video/live) and GROUP (CI/NoCI). Analysis revealed significant main effect of AUDIO $\left(F_{(1,95)}=70.60, p<.001, \eta^{2} p=.43\right)$, participants' taps being more consistent in metronome than in music conditions. There was also a significant main effect of SOCIAL $\operatorname{CONTEXT}\left(F_{(1,95)}=29.48, p<.001, \quad \eta^{2} p=.24\right)$, participants' taps being more consistent in video than in live conditions. Finally, there was a significant AUDIO by SOCIAL CONTEXT interaction $\left(F_{(1,95)}=32.44, p<.001\right.$, $\left.\eta^{2} p=.25\right)$. When synchronizing with metronome beats, participants' taps were more constant in the video condition than in the live condition as confirmed by posthoc test with Bonferroni correction for multiple comparisons $(p<.001)$, which was not the case when synchronizing with musical beats (see Figure 4.A). There was no significant GROUP effect or any other significant interactions (see Figure 4.C).

SMS Accuracy. We conducted an ANOVA on SMS accuracy with the factors AUDIO (metronome/music), SOCIAL CONTEXT (video/live) and GROUP ((CI/NoCI). Analysis revealed significant main effects of the AUDIO $\left(F_{(1,95)}=134.6, p<.001, \eta^{2} p=.59\right)$, participants' taps being more anticipated in the metronome condition than in the music condition. There was also a significant main effect of the SOCIAL CONTEXT $\left(F_{(1,95)}\right.$ $\left.=18.09, p<.001, \eta^{2} p=.16\right)$, participants' taps being more anticipated in the video condition than in the live condition. Finally they was a significant AUDIO by SOCIAL CONTEXT interaction $\left(F_{(1,95)}=49.58, p<.001\right.$, $\left.\eta^{2} p=.34\right)$. When synchronizing with metronome beats, participants' taps were more anticipated in the video condition than in the live condition, as confirmed by posthoc test with Bonferroni correction for multiple comparisons $(p<.001)$, whereas when synchronizing with music (see Figure 4.B), the accuracy was not affected by the social context $(p>.05)$ with taps being slightly delayed in both live and video conditions. There was no significant GROUP effect or any other significant interactions (see Figure 4.D).

To verify whether or not singing during the music conditions interfered with SMS scores, we tested the correlations between duration of lip movements during singing and the two SMS measures (consistency and accuracy). The results showed no correlation in the live and video conditions with music (all ps $>0.05$ ) suggesting that singing activities did not disrupt SMS scores. 


\section{Musician's performances}

We analyzed the musician's synchronization with the auditory sequences in the live condition. The musician's mean tapping rate (mean ITI) was significantly smaller (more synchronous) in the metronome than in the musical condition (Wilcoxon signed-rank test: $Z=8.21, p<.001$ ), although his synchronization to the auditory sequences was highly accurate in both conditions $(<5 \mathrm{~ms})$. Moreover, the musician's tapping variability (CV of the ITI) was significantly smaller in the metronome than the musical condition (Wilcoxon signed-rank test: $Z=7.43, p$ $<.001)$. Table 2 indicates the musician's performances in the live and video conditions.

\section{Discussion}

The aim of this study was to evaluate the impact of the social context and of the auditory conditions on SMS abilities in two groups of elderly patients with or without cognitive impairments, at the time of testing. SMS was measured during a hand-tapping task in response to beats in metronomic or musical sequences, with a live or video presence of a musician. As expected, participants were better at synchronizing to metronome than to music with their taps being more consistent and more anticipated. We found that the effect of social context varied as a function of the auditory conditions. Although participants were better at synchronizing with a metronome beat when facing a pre-recorded video of a musician rather than a live performance, the social context had no effect on SMS in the musical condition. Furthermore, we did not observe differences between the two patient groups in any SMS scores.

Participants' SMS was more consistent and anticipated in the metronome than in the musical conditions. To ensure that these results cannot be explained by the musician's performance, we examined his SMS scores. As in the patients, they were better with the metronome than with the music. However, the difference between the musician's synchronization and the auditory beats in these two conditions was too small to explain the observed effect on patients' synchronization and to suggest that the musician adapted his performance to the patient's taps. Participants' decline in SMS performance in the musical condition reflects the complexity of synchronizing to beats in music. Indeed, SMS with a musical sequence requires extracting and anticipating the strong beats in a melody. Such synchronization is therefore more difficult to achieve than synchronization with a regular metronomic sequence composed exclusively of strong beats. This pattern of results, which has been documented in literature in healthy young adults $[26,40]$ and in individuals with Parkinson's disease [41], also extends to a general aged population.

Interestingly, we found that SMS performances with metronome and music were differently impacted by the social context. In other words, when the participant tapped to a metronome in front of a live musician, consistency and anticipation decreased, as compared with a video of the musician. Such social effects were not obtained when participants synchronized taps to music. In the metronome conditions, participants' SMS performance from the present study was better when facing a video of a musician, rather a live presence. This result seems counterintuitive as studies on young adults support the idea that interpersonal interaction improves SMS with metronome [20] and also with music [18]. However, the present result is congruent with data we obtained in a group of patients with moderate to severe AD [15]. The deterioration in SMS with live performance may be explained due to the cognitive load of synchronizing to the auditory stimuli competing with that of synchronizing to the musician's movements [42]. However, this explanation is unlikely considering the high temporal precision and regularity with which the musician synchronized with the auditory sequences. An alternative explanation is that the SMS performance decreased in the live condition because of the mere presence of an observer. Audience effects refer to the social influence of having another individual present during a task [43]. As reported in social psychology, the sole presence of an observer induces social pressure and stress, which can positively or negatively modulate an individual's performance in a task [44-46]. In our study, we hypothesized that live presence of the musician may improve participants' performances by motivating them and by focusing their attention on the tapping task. On the contrary, it seems that live presence deteriorated participants' performances by dividing their attention. Whatever the underlying mechanism may be, increased cognitive load or existence of social pressure, the physical presence of another individual deteriorated performance in this task. The aforementioned effect was not dependent on participants' group, suggesting that this response pattern is not specific to the neurodegenerative diseases investigated here $(\mathrm{AD}$, vascular and mixed dementia). Assessing participants' attention and social cognition (social anxiety, empathy) may allow a better understanding of this phenomenon in the future.

Unlike the metronome condition, the social context had no impact on participants SMS in the musical condition, the taps being delayed in response to the musical beat. Several hypotheses can be proposed to explain this difference. First, synchronizing with a musical beat is more difficult than synchronizing with a metronome one, and account for why SMS scores were globally less consistent and less anticipated (and even slightly delayed) in music condition. Tapping variability in the musical condition may have flattened the differences associated with social context. Second, music is a pleasant stimulus that can modulate the emotional state and increase social sharing of experiences. The stress induced by a live social presence, leading to performance deterioration in the metronome condition, may be mitigated when synchronizing to music due to its positive emotional impact. Furthermore, moving to music with another individual is a pleasant and ecological social task, close to a real life situation $[47,48]$. Thus, the musical 
condition might be more natural and enjoyable than the metronomic one, diminishing the audience effect.

In contrast to our expectations, we found no differences in rhythmic performances between the CI and the NoCI groups, be it in the spontaneous tapping task or in the SMS task. It is noteworthy that the spontaneous motor tempo (ITI of $800 \mathrm{~ms}$ or less, corresponding to $75 \mathrm{bpm}$ or more) obtained in these patients is consistent with data previously reported in patients with AD [49], and slower than the tempo generally observed in healthy elderly people [50]. This result also confirmed that the tempo that we chose in this study for the SMS task (IOI of $800 \mathrm{~ms}$, or $75 \mathrm{bpm}$ ) was appropriate for this patient population. The lack of group difference in SMS performance may be slightly surprising for some, as previous studies have reported rhythmic deficits in AD using continuous tapping tasks [24,25]. However, in these tasks, participants needed to continue reproducing a beat without the help of external stimuli, which is more cognitively demanding in terms of attentional resources and working memory than the SMS task used in our study. Our present results suggest that SMS abilities are comparable in patients with or without cognitive impairments, despite a significant cognitive and behavioral decline in the CI group. As evidenced by neuroimaging studies [51-53], the neural correlates of synchronization and audio-motor coupling include the pre-motor cortex, the pre-supplementary motor area, the basal ganglia and the cerebellum. It might therefore be possible that these regions are relatively preserved even in moderate $\mathrm{AD}$; however we approach this interpretation cautiously since about three quarters of the patients in the CI group presented a mixed or a vascular dementia with different cerebral dysfunction. Even though the participants of the CI group were impaired as compared to participants of the NoCI group in terms of cognitive (MMSE [36]) and behavioral measures (ADL score [35]), we cannot be sure that patients in the NoCI group were all healthy, given that for many the hospital visit was instigated by a recent fall. Motor difficulties and particularly those related to walking and falls can be a predictor for the onset of Parkinson's disease and even AD [54-56]. We have therefore no guarantee that the NoCI group were not at a prodromal stage of a degenerative disease. Nevertheless, we can be quite confident that these matched participants from the NoCI group presented fewer cognitive and behavioral deficits in comparison with patients from the CI group. To better document this question, further research needs to be carried out to evaluate SMS abilities in healthy older adults but also in patients at several time-points during their disease progression.

To conclude, our results show that it is possible to collect well-controlled measurements of SMS in elderly people with or without cognitive deficits. Although experimental rigor is often difficult to reconcile with the constraints of clinical reality, particularly in the field of pathological ageing, this experimental paradigm based on a hand-tapping task performed with a partner as part of a musical task, seems suitable for evaluating SMS. Thus, this study validates an experimental device consisting of a chair equipped with a tablet to record SMS in patients with impaired cognitive faculties (decreased attention, motor, motivation and communication disorders). This paradigm is also original in its examination of the impact of social interaction induced by a live partner compared to a virtual presence. By showing that it is possible to encourage a patient to synchronize with others, even in the presence of a video recording, our study provides a relevant measure to predict clinical effects that might used as outcome measures in future studies. It also opens up promising therapeutic potential for patients living at home, in remote areas or in confinement. Mobile technology might be used to train patients. By synchronizing movements to music and engaging patients in rhythmic action with others, cognitive-motor skills and temporal tracking mechanisms involved in verbal and non-verbal interaction would be stimulated. Stimulating SMS and musical entrainment in patients might increase the alignment of internal oscillations between brain regions with that of beats in auditory rhythms. By progressively acting in synchrony with an environmental system (musical rhythm) through the coupling of perception and action, we propose that cognition and social abilities will improve through the use of rhythmic stimulation in elderly. We therefore predict that such trainings will better the action-perception coupling and interpersonal coordination, consequently boosting verbal and non-verbal communicative skills. This remains to be verified in the future. The idea is not to replace musical activities performed in the presence of others with a virtual interaction, but rather to complement classical interventions. Virtual initiatives, via tele-health and similar platforms, are an unexplored cost-effective and easy to implement tool to address everyday social isolation experienced by this geriatric population. Being able to employ video recordings and mobile technology may allow healthcare providers to circumvent practical difficulties (i.e. rural and low-resource settings, or other exceptional situation such as the current pandemic) that may act as a barrier to providing care that has historically been considered niche or resource intensive.

\section{Acknowledgements}

This research was carried out with the support of the Conseil Régional des Hauts-de-France and the University of Lille at M.G., the Ministry of Foreign Affairs (Hubert Curien partnership) at S.S. and M.L. as well as the France Alzheimer and Institut Universitaire de France at S.S. This research was carried out in cooperation with the Department of Musicology of Ghent University, and financed by the project "Expressive Music Interaction" which is financially supported by the Flemish government (Methusalem grant). This research was also supported by the Globalink Research Award from Mitacs Canada to A.S. The authors would also like to thank Ivan Schepers of Ghent University for the development of the material. We are especially grateful to Sotirios Sideris, the musician who participated in the study, to the geriatrician Dr. J. Roche, to the psychologists C. Jougleux, S. Schoenenburg 
and A. Clerckx, to the staff of the Bateliers day hospital at Lille, and to the participants who agreed to participate in this study.

\section{Conflict of interest}

The authors have no conflict of interest to report.

\section{Acknowledgements}

The authors would like to thank Ivan Schepers of Ghent University for the material development. We are especially grateful to the musician who participated in the study Sotirios Sideris, to the geriatrician Dr. J. Roche, to the psychologists C. Jougleux, S. Schoenenburg and A. Clerckx, to the staff of the Bateliers day hospital at Lille, and to the participants who agreed to participate in this study.

\section{References}

[1] Hodges JR (2000) Memory in the dementias. In The Oxford handbook of memory, pp. 441-459.

[2] Feyereisen P, Hupet M (2007) Pragmatic skills in the early stages of Alzheimer's disease : an analysis by means of a referential communication task. Int. J. Lang. Commun. Disord. 42, 1-17.

[3] Potkins D, Myint P, Bannister C, Tadros G, Chithramohan R, Swann A, O'Brien J, Fossey J, George E, Ballard C, MargalloLana M (2003) Language impairment in dementia: Impact on symptoms and care needs in residential homes. Int. J. Geriatr. Psychiatry 18, 1002-1006.

[4] Bediou B, Ryff I, Mercier B, Milliery M, Hanaff MA D'Amato T, Bonnefoy M, Vighetto A, Krolak-Salmon P (2009) Impaired social cognition in mild alzheimer disease. $J$. Geriatr. Psychiatry Neurol. 22, 130-140.

[5] National Institute for Health and Care Excellence (2018) Dementia: Assessment, management and support for people living with dementia and their carers.

[6] Sihvonen AJ, Särkämö T, Leo V, Tervaniemi M, Altenmüller E, Soinila S (2017) Music-based interventions in neurological rehabilitation. Lancet Neurol. 16, 648-660.

[7] van der Steen JT, Van Soest-Poortvliet MC, Van Der Wouden JC, Bruinsma MS, Scholten RJ, Vink AC (2018) Music-based therapeutic interventions for people with dementia (Review). Cochrane Database Syst. Rev. 7,

[8] Narme P, Clément S, Ehrlé N, Schiaratura L, Vachez S, Courtaigne B, Munsch F, Samson S (2014) Efficacy of musical intervention in dementia: evidence from a randomized controlled trial. J. Alzheimer's Dis. 38, 31-42.

[9] Choi AN, Lee MS, Cheong KJ, Lee JS (2009) Effects of group music intervention on behavioral and psychological symptoms in patients with dementia: A pilot-controlled trial. Int. J. Neurosci. 119, 471-481.

[10] Sakamoto M, Ando H, Tsutou A (2013) Comparing the effects of different individualized music interventions for elderly individuals with severe dementia. Int. Psychogeriatrics 25, 775-784.

[11] Särkämö T, Tervaniemi M, Laitinen S, Numminen A, Kurki M, Johnson JK, Rantanen P, Julene K, Rantanen P (2014) Cognitive, emotional, and social benefits of regular musical activities in early dementia: Randomized controlled study. Gerontologist 54, 634-650.

[12] Sherratt K, Thornton A, Hatton C (2004) Music interventions for people with dementia: A review of the literature. Aging
Ment. Heal. 8, 3-12.

[13] Holmes C, Knights A, Dean C, Hodkinson S, Hopkins V (2006) Keep music live: music and the alleviation of apathy in dementia subjects. Int. Psychogeriatrics 18, 623-630.

[14] Lesaffre M, Moens B, Desmet F (2017) Monitoring music and movement interaction in people with dementia. In The Routledge Companion to Embodied Music Interaction Routledge, pp. 294-303.

[15] Ghilain M, Hobeika L, Schiaratura L, Lesaffre M, Six J, Desmet F, Clément S, Samson S (2020) Synchronisation sensorimotrice et comportements non verbaux dans la maladie d'Alzheimer : l'influence du contexte social et musical. Geriatr. Psychol. Neuropsychiatr. Vieil. 18, 213-222.

[16] Oullier O, Guzman GC De, Jantzen KJ, Lagarde J, Kelso JAS (2008) Social coordination dynamics : Measuring human bonding. Soc. Neurosci. 3, 178-192.

[17] van Ulzen NR, Lamoth CJC, Daffertshofer A, Semin GR, Beek PJ (2008) Characteristics of instructed and uninstructed interpersonal coordination while walking side-by-side. Neurosci. Lett. 432, 88-93.

[18] Demos AP, Carter DJ, Wanderley MM, Palmer C (2017) The unresponsive partner : roles of social status, auditory feedback , and animacy in coordination of joint music performance. Front. Psychol. 8, 149

[19] Kirschner S, Tomasello M (2009) Joint drumming: Social context facilitates synchronization in preschool children. $J$. Exp. Child Psychol. 102, 299-314.

[20] Konvalinka I, Vuust P, Roepstorff A, Frith CD (2010) Follow you, follow me: continuous mutual prediction and adaptation in joint tapping. Q. J. Exp. Psychol. (Hove). 63, 2220-30.

[21] Repp BH, Su Y-H (2013) Sensorimotor synchronization: a review of recent research (2006-2012). Psychon. Bull. Rev. 20, 403-52.

[22] Kluger A, Gianutsos JG, Golomb J, Ferris SH, Reisberg B (1997) Motor/psychomotor dysfunction in normal aging, mild cognitive decline, and early alzheimer's disease: diagnostic and differential diagnostic features. Int. Psychogeriatrics 9, 307316.

[23] Yan JH, Rountree S, Massman P, Doody RS, Li H (2008) Alzheimer's disease and mild cognitive impairment deteriorate fine movement control. J. Psychiatr. Res. 42, 1203-1212.

[24] Martin E, Blais M, Albaret J, Pariente J, Tallet J (2017) Human movement science alteration of rhythmic unimanual tapping and anti-phase bimanual coordination in Alzheimer's disease : a sign of inter-hemispheric disconnection? Hum. Mov. Sci. 55, 43-53.

[25] Bangert AS, Balota DA (2012) Keep up the pace: declines in simple repetitive timing differentiate healthy aging from the earliest stages of Alzheimer's disease. J. Int. Neuropsychol. Soc. 18, 1052-1063.

[26] Aschersleben G (2002) Temporal control of movements in sensorimotor synchronization. Brain Cogn. 48, 66-79.

[27] Dalla Bella S, Farrugia N, Benoit C, Begel V, Verga L, Harding E, Kotz SA (2016) BAASTA : Battery for the Assessment of Auditory Sensorimotor and Timing Abilities. Behav. Res. Methods 49, 1128-1145.

[28] Bienkiewicz MMN, Craig CM (2015) Parkinson's is time on your side? Evidence for difficulties with sensorimotor synchronization. Front. Neurol. 6, 249.

[29] Cock VC De, Dotov DG, Ihalainen P, Bégel V, Galtier F, Lebrun C, Picot MC, Driss V, Landragin N, Geny C, Dalla Bella S (2018) Rhythmic abilities and musical training in Parkinson's disease : do they help ? NPJ Park. Dis. 4, 1-8.

[30] Merchant H, Luciana M, Hooper C, Majestic S, Tuite P (2008) Interval timing and Parkinson's disease : heterogeneity in temporal performance. Exp. Brain 184, 233-248.

[31] American Psychiatric Association (2013) Diagnostic and statistical manual of mental disorders (DSM-5®), American Psychiatric Pub.

[32] Poitrenaud J (2001) Evaluation du niveau socio-culturel. In La consultation de gériatrie, Masson. P., ed., pp. 8-9.

[33] Ehrlé N (1998) Traitement temporel de l'information auditive et temporal.

[34] Spielberger CD, Gorsuch R, Lushene RE, Vagg PR, Jacobs GA (1983) Manual for the state-trait anxiety inventory. Palo Alto, 
CA Consult. Psychol. Press.

[35] Galasko D, Bennett D, Sano M, Ernesto C, Thomas R, Grundman M, Ferris S, Study the ADC (1997) An Inventory to Assess Activities of Daily Living for Clinical Trials in Alzheimer's Disease. Alzheimer Dis. Assoc. Disord. 11,

[36] Folstein MF, Folstein SE, McHugh PR (1975) A practical state method for grading the cognitive state of patients for the clinician. J. Psychiatr. Res. 12, 189-198.

[37] Fisher NI (1995) Statistical analysis of circular data, Cambridge University Press.

[38] Berens P (2009) CircStat: a MATLAB toolbox for circular statistics. J. Stat. Softw. 31,

[39] Wilkie D (1983) Rayleigh Test for Randomness of Circular Data. Appl. Stat. 32, 311-312.

[40] Repp BH (2005) Sensorimotor synchronization : A review of the tapping literature. Psychon. Bull. Rev. 12, 969-992.

[41] Benoit C, Bella SD, Farrugia N, Obrig H, Mainka S, Kotz SA (2014) Musically cued gait-training improves both perceptual and motor timing in Parkinson's disease. Front. Hum. Neurosci. 8, 1-11.

[42] Demos AP, Chaffin R, Begosh KT, Daniels JR, Marsh KL (2012) Rocking to the beat: effects of music and partner's movements on spontaneous interpersonal coordination. J. Exp. Psychol. Gen. 141, 49-53.

[43] Zajonc RB (1965) Social facilitation. Science (80-. ). 149, 269 274.

[44] Welford AT (1973) Stress and performance. Ergonomics 16, 567-580.

[45] Bray RM, Sugarman R (1980) Social facilitation among interacting groups: Evidence for the Evaluation-Apprehension Hypothesis. Personal. Soc. Psychol. Bull. 6, 137-142.

[46] McKinney ME, Gatchel RJ, Paulus PB (1983) The Effects of Audience Size on High and Low Speech-Anxious Subjects During an Actual Speaking Task. Basic Appl. Soc. Psych. 4, $73-87$.
[47] Wiltermuth SS, Heath C (2009) Synchrony and cooperation. Psychol. Sci. 20, 1-5.

[48] Ehrenreich B (2007) Dancing in the streets: A history of collective joy, New York: Metropolitan Books.

[49] Rabinowitz I, Lavner Y (2014) Association between finger tapping, attention, memory, and cognitive diagnosis in elderly patients. Percept. Mot. Skills 119, 259-278.

[50] McAuley JD, Jones MR, Holub S, Johnston HM, Miller NS (2006) The time of our lives : life span development of timing and event tracking. J. Exp. Psychol. Gen. 135, 348-367.

[51] King JB, Jones KG, Goldeberg E, Rollins M, MacNamee K, Moffit C, Naidu SR, Ferguson MA, Garcia-Leavitt E, Amaro J, Breitenbach KR, Watson JM, Gurgel RK, Anderson JS, Foster NL (2019) Increased Functional Connectivity After Listening to Favored Music in Adults With Alzheimer Dementia. J. Prev. Alzheimer's Dis. 6, 56-62.

[52] Jacobsen J-H, Stelzer J, Fritz TH, Chételat G, La Joie R, Turner $\mathrm{R}$ (2015) Why musical memory can be preserved in advanced Alzheimer' s disease. Brain 138, 2438-2450.

[53] Zatorre RJ, Chen JL, Penhune VB (2007) When the brain plays music: Auditory-motor interactions in music perception and production. Nat. Rev. Neurosci. 8, 547-558.

[54] Belghali M, Chastan N, Cignetti F, Davenne D, Decker LM (2017) Loss of gait control assessed by cognitive-motor dualtasks : pros and cons in detecting people at risk of developing Alzheimer' s and Parkinson' s diseases. GeroScience 39, 305329.

[55] Buracchio T, Dodge HH, Howieson D, Wasserman D, Kaye J (2010) The Trajectory of Gait Speed Preceding Mild Cognitive Impairment. Arch Neurol 67, 980-986.

[56] Verghese J, Wang C, Lipton RB, Holtzer R, Xue X (2007) Quantitative gait dysfunction and risk of cognitive decline and dementia. J Neurol Neurosurg Psychiatry 78, 929-935. 
Figure 1: Experimental set-up. (A) Participant performed the SMS task in front of a musician, by tapping on a tablet fixed on the chair right armrest. The musician was present either as (B) a life size recorded broadcast on a screen (video condition), or (C) a live performance (live condition)

A.

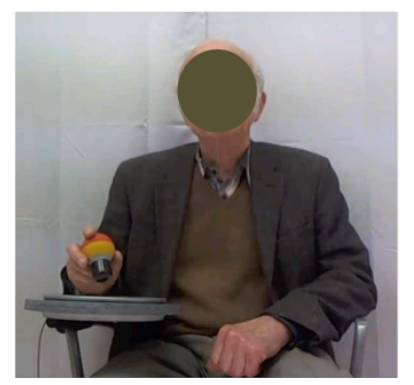

B.

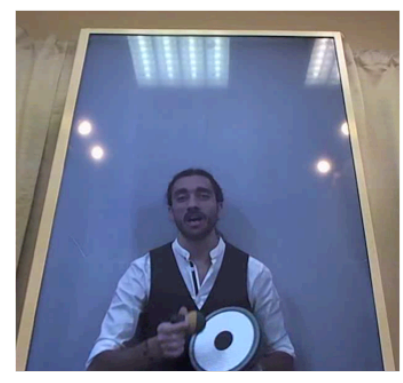

c.

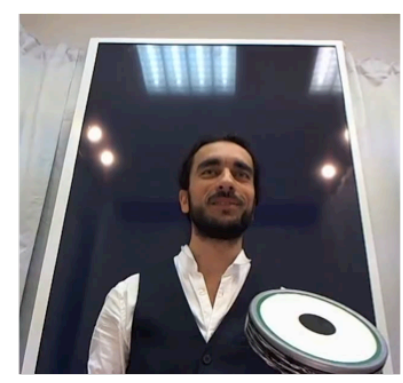

Figure 2. Example of synchronization analysis of an experimental trial. Each tap is represented as a blue dot on the trigonometric circle. Tap position depends on it's time delay in relation to the beat occurrence. with A negative angle indicates if the tap occurred before the beat (angle between $-180^{\circ}$ and 0 ), and a positive angle is present if the tap occurred after the beat (angle between 0 and $+180^{\circ}$ ). 0 corresponds to a perfect match between the tap and the beat, whereas $-180^{\circ} / 180^{\circ}$ corresponds to a tap at the antiphase of the beat. The mean resultant vector $\vec{R}$ represents participant's synchronization performance: $\vec{R}$ length gives participant consistency, and $\vec{R}$ angle with the vertical axis $\theta$ represents participant accuracy.

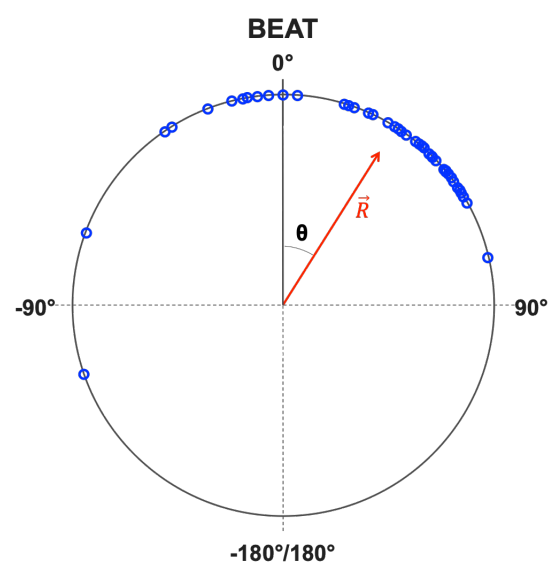

Figure 3: Spontaneous tapping task. This figure reports the mean inter-tap interval (ITI) ( \pm SEM) and the mean coefficient of variation $(\mathrm{CV})( \pm$ SEM) of the CI group and the NoCI group. No significant difference was found between groups. n.s. $=$ non-significant
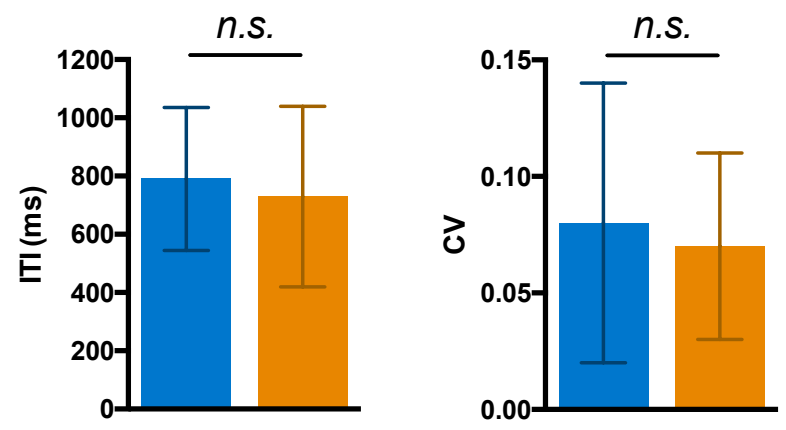

NoCl group $(\mathrm{N}=49)$

Cl group $(\mathrm{N}=48)$ 
Figure 4: Analysis of SMS consistency and accuracy. This figure reports mean synchronization consistency ( \pm SEM) and accuracy $( \pm$ SEM) depending on the auditory sequence (metronome or music) and the social context (video or live) (panel $\mathbf{A}$ and B), and depending on the participant group (panel C and D). Consistency can vary between 0 (random distribution of taps) and 1 (perfect regularity of taps). Negative accuracy values indicate taps occurring before the beat (anticipated), whereas positive accuracy values indicate taps occurring after the beat (delayed). Participants' consistency was better with more anticipation when synchronizing with a metronome beat than a musical one. Moreover, when participants tapped with metronome beats, their consistency was better (panel A) and their anticipation larger (panel B) with a video presence than with a live presence of a partner. When participants tapped with musical beats, the social context did not impact their consistency and accuracy. No significant difference was observed between the CI and the NoCI groups on the consistency (panel C) and the accuracy (panel D). n.s.=non-significant

A

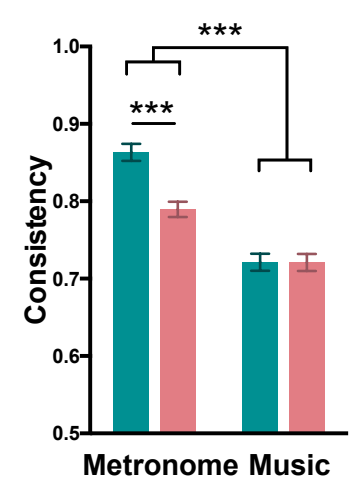

C

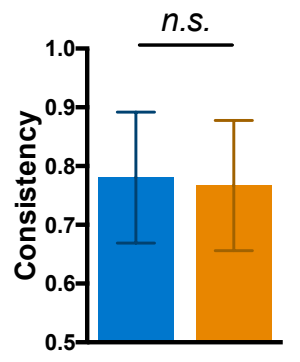

B

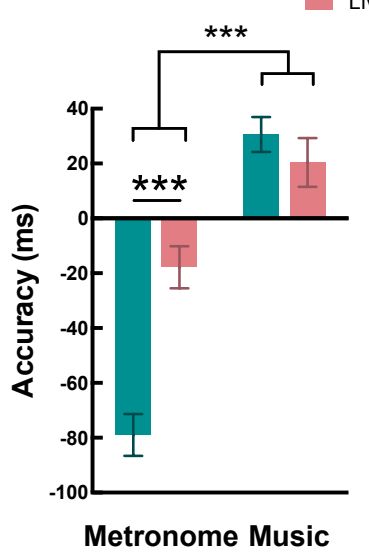

D

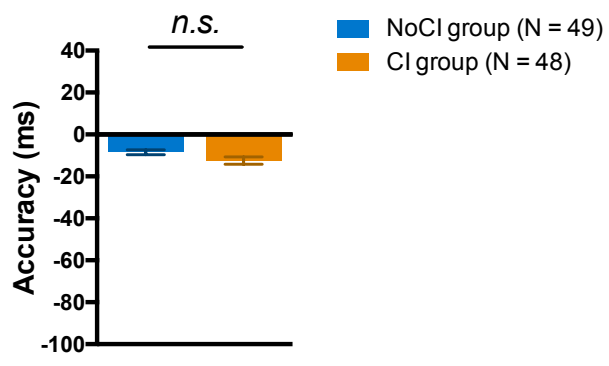


Table 1. Demographic and clinical characteristics (mean \pm std) of patients with cognitive impairments (CI) and patients without cognitive impairment (NoCI).

\begin{tabular}{lccc}
\hline & CI $(\boldsymbol{N}=\mathbf{4 8})$ & NoCI $(\boldsymbol{N}=\mathbf{4 9})$ & .08 \\
\hline Age & $82.8 \pm 5.5$ & $80.9 \pm 5.2$ & .66 \\
Gender (female/male) & $38 / 10$ & $37 / 12$ & .34 \\
Socio-cultural level (4 levels) ${ }^{\mathrm{a}}[32]$ & $8 / 26 / 6 / 8$ & $7 / 19 / 9 / 14$ & .46 \\
Musical expertise questionnaire (/28) [33] & $4.3 \pm 3.1^{\mathrm{b}}$ & $4.8 \pm 3.2$ & .08 \\
STAI (State-Trait Anxiety Inventory) (/40) [34] & $29.4 \pm 10.8^{\mathrm{b}}$ & $33.2 \pm 12.3$ & \\
ADL (Activities of Daily Living) (/6) [35] & $5.0 \pm 0.9^{\mathrm{b}}$ & $5.7 \pm 0.6$ & $\mathbf{0 0 0 1}$ \\
MMSE (Mini-Mental Scale Examination) (/30) [36] & $20.2 \pm 3.6^{\mathrm{b}}$ & $28.1 \pm 1.2$ & $\mathbf{0 0 0 1}$
\end{tabular}

${ }^{a}$ Socio-cultural level evaluated according to 4 levels. Level 1 is the lowest, corresponding to the absence of diploma. Level 4 is the highest, corresponding to a high school diploma

${ }^{\mathrm{b}}$ missing data for one participant

Table 2. Musician's performances. Mean hand-tapping rate (mean inter-tap interval, ITI) ( \pm SEM) and mean tapping variability (coefficient of variation $(\mathrm{CV})$ of the ITI) $( \pm$ SEM) of the musician in both auditory (metronome/musical) and social conditions (video/live).

\begin{tabular}{lccc}
\hline & & $\begin{array}{c}\text { Inter-tap interval (ITI) } \\
(\mathbf{m s})\end{array}$ & $\begin{array}{c}\text { Coefficient of variation }(\mathbf{C V}) \\
(\mathbf{m s})\end{array}$ \\
\hline Metronome & Video & -.13 & .30 \\
& Live & $.57 \pm .15$ & $.90 \pm .031$ \\
Music & Video & 3.81 & 1.1 \\
& Live & $4.35 . \pm .16$ & $1.1 \pm .18$ \\
\hline
\end{tabular}

